\section{ORIGINAL RESEARCH}
S.K. Handique
R.R. Das
B. Saharia
P. Das

R. Buragohain

P. Saikia

\title{
Coinfection of Japanese Encephalitis with Neurocysticercosis: An Imaging Study
}

BACKGROUND AND PURPOSE: Coinfection of neurocysticercosis (NCC) and Japanese encephalitis (JE) has been advocated as more than a chance occurrence resulting in poor outcome. We undertook this study to determine whether the association of the 2 infections is more than a chance occurrence, to define the imaging characteristics of coinfections, and to explore the synergistic effect of NCC in JE.

MATERIALS AND METHODS: Sixty-two patients with JE were studied by MR imaging and CT. CT was done in 53 and MR imaging in 53 patients. The diagnosis of JE was established by CSF JE virus immunoglobulin M capture (MAC) enzyme-linked immunosorbent assay (ELISA). NCC was diagnosed from imaging. A control group of 385 patients was evaluated by imaging for prevalence of NCC in the general population.

RESULTS: A significantly high association of NCC with JE (19.3\%) was observed in comparison with prevalence of NCC in control subjects $(1.04 \% ; P=.0003)$. JE lesions in coinfection were significantly asymmetric with lateralization to the side of the brain having the maximum NCC or a cyst with edema. The JE lesions in coinfections were more florid, with a significantly higher proportion of abnormal CT scans and more abnormal MR imaging. Coinfections were significantly more common in children. Significantly lower CSF MAC-ELISA units in patients with coinfection reflected low CSF IgM levels, suggesting altered immune status.

CONCLUSION: In our series, there was a strong association between JE and NCC, and, thus, this coinfection was more than a chance occurrence.

J apanese encephalitis (JE) is a mosquito-borne viral encephalitis that is endemic to several tropical and semitropical countries in Asia. A total of 50,000 new cases occur annually across the endemic regions of the world, of which 10,000 people die. JE is endemic in India, with outbreaks reported in several Indian states in the past decades. Pigs act as amplifier of the JE virus (JEV). Epidemics occur in the summer rainy season in India when mosquitoes are abundant. Clinically, the disease manifests as fever, headache, and altered sensorium with or without focal neurologic symptoms. ${ }^{1}$ On CT and MR imaging, JE lesions are most commonly seen in the thalami. Other areas involved are the substantia nigra, basal ganglia, cerebral cortex, hippocampus, pons, midbrain, medulla, cerebellum, and white matter. ${ }^{2-4}$

Neurocysticercosis (NCC) is caused by the larval stage of the pork tapeworm, Taenia solium and results from the ingestion of the eggs of the adult tapeworm by the fecal-oral route. It is endemic to and a public health problem in India. Neuroimaging by CT or MR imaging is useful for establishing the diagnosis. ${ }^{5}$

Coinfection of JE and NCC has been reported in China and India. ${ }^{6-8}$ Although both diseases have common epidemiologic and sociodemographic factors like pig rearing, poor socioeconomic status, hygienic conditions, malnutrition, and so forth, more than a casual association of the 2 conditions has been suggested. The presence of NCC has been advocated as a prognosticator of poor outcome in JE. ${ }^{6,7}$ Few imaging studies have studied this coinfection. ${ }^{9,10}$ These have drawn conflicting con-

Received April 9, 2007; accepted after revision June 13.

From the Departments of Radiology and Imaging (S.K.H., B.S., P.D., R.B.), Neurology (R.R.D.), and Microbiology (P.S.), Institute of Neurological Sciences, Dispur, Assam, India.

Please address correspondence to Sanjeev K. Handique, Department of Radiology and Imaging, Institute of Neurological Sciences, Dispur-781006, Assam, India; e-mail: sanjeevhandique@hotmail.com or sanjeev_handique@yahoo.co.in

三E Indicates article with supplemental on-line tables.

DOI 10.3174/ajnr.A0769 clusions. Singh et $\mathrm{al}^{9}$ reported the imaging features in coinfection but did not compare with a cohort of JE patients without coinfection. Azad et $\mathrm{al}^{10}$ found an increased prevalence of NCC in patients with JE but did not find any statistically significant difference from the prevalence of NCC in control subjects. We undertook this study to find out if there is a significant association of JE with NCC, to define the imaging characteristics of coinfection of JE and NCC, to compare them with a cohort of JE subjects without coinfections, and to explore the synergistic effect of NCC in JE.

\section{Materials and Methods}

Sixty-two consecutive patients with JE underwent CT or MR imaging scans in the period of June 2000 through August 2003 as part of a prospective study, part of the results of which have been published earlier. ${ }^{4} \mathrm{MR}$ imaging was done in 53 and CT in 53 subjects. Both CT and MR imaging were done in 44 subjects; MR imaging or CT was each done in only 9 subjects. Our patients were from the 7 Northeast Indian states and came from a diverse rural, semirural, and urban background. There were 45 males and 17 females aged 9 months to 75 years. Fever and altered sensorium were the most common presenting features seen in all of the patients. Seizures and focal neurologic symptoms were present in some. All of the patients had a routine blood and CSF examination. The diagnosis of JE was established by positive CSF JEV immunoglobulin M (IgM) antibody capture enzyme-linked immunosorbent assay (MAC-ELISA). All of the patients had MACELISA units $\geq 100$, which were taken as definite for JE. ${ }^{11}$

MR imaging of the head was done in a $1 \mathrm{~T}$ scanner. T1-weighted (TR, $600 \mathrm{~ms} / \mathrm{TE}, 15 \mathrm{~ms}$ ), T2-weighted (TR, $3500 \mathrm{~ms} / \mathrm{TE}, 90 \mathrm{~ms}$ ), and proton attenuation (TR, $1700 \mathrm{~ms} / \mathrm{TE}, 17 \mathrm{~ms}$ ) images were obtained in axial, coronal, and sagittal planes. One patient with coinfection had contrast-enhanced MR imaging, whereas the rest had plain MR imaging only. Fourteen patients ( 4 with coinfection of JE and NCC) had repeat MR imaging on clinical improvement of JE symptoms. Plain or contrast-enhanced CT was done in a conventional CT scanner using 

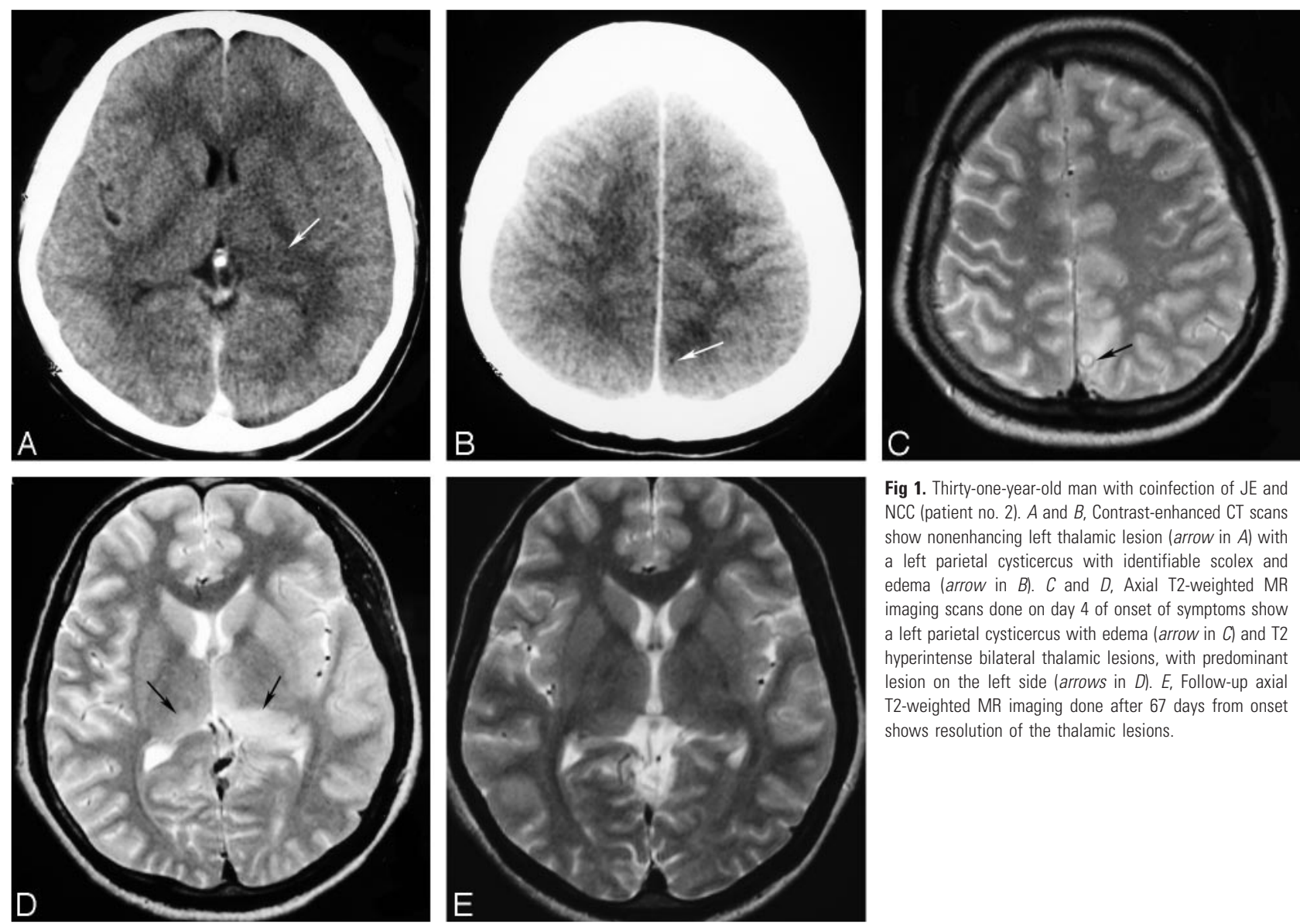

Fig 1. Thirty-one-year-old man with coinfection of JE and NCC (patient no. 2). $A$ and $B$, Contrast-enhanced CT scans show nonenhancing left thalamic lesion (arrow in $A$ ) with a left parietal cysticercus with identifiable scolex and edema (arrow in B). C and D, Axial T2-weighted MR imaging scans done on day 4 of onset of symptoms show a left parietal cysticercus with edema (arrow in $C$ ) and T2 hyperintense bilateral thalamic lesions, with predominant lesion on the left side (arrows in D). E, Follow-up axia T2-weighted MR imaging done after 67 days from onset shows resolution of the thalamic lesions.

5- or 10-mm axial sections. Two patients (1 with NCC) had repeat plain CT examination.

The diagnosis of NCC was made on neuroimaging findings alone as per well-described imaging criteria. ${ }^{12}$ CSF cysticercus immunoglobulin G ELISA was done in 4 patients with NCC but was found to be negative. Cysticerci were counted and evaluated for the presence of perilesional edema. The presence, signal intensity, or attenuation and distribution of JE lesions was assessed in the scans.

A control group of patients scanned in our institute for indications other than seizures was prospectively evaluated for NCC prevalence in the population studied. Patients with mild head trauma, headache, psychiatric complaints, giddiness, and so forth who had more or less normal scans with good assessable brains were included. The prevalence of seizures in India is estimated at $0.4 \%-0.6 \%$, and $34.6 \%$ of all seizures in India are due to NCC. ${ }^{13,14}$ As a tertiary neurocare hospital, $10 \%$ of our patients are scanned due to seizures. $\mathrm{Pa}-$ tients with seizures were, therefore, excluded from the control group to remove bias. MR imaging protocols were the same as above. A 16-section multidetector CT was used to scan these patients. The population of Northeast India being approximately 38.5 million, a sample size calculator (Raosoft, Seattle, Wash) was used to define the size of the control group for a 95\% confidence level and a 5\% margin of error. A total of 385 patients were scanned in all, with CT in 342 and MR imaging in 43 patients. There were 261 males and 124 females of ages 2-82 years. Contrast examination was done in $31 \mathrm{CT}$ and $4 \mathrm{MR}$ imaging patients. The presence of NCC was evaluated in these.

Statistical analysis of data was done by $X^{2}$ test. Our institute's ethics committee approved this work.

\section{Results}

Twelve patients (19.3\%) with JE showed coinfection with NCC. Eleven patients had NCC on MR imaging. In 1 patient (patient 12), NCC was diagnosed from CT. Of the 12 patients with NCC, 7 had single NCC, 1 had 2 cysts, and 4 had multiple NCC (patient 12 had multiple bilateral NCC on CT; on-line Tables 1 and 2). In the control group, 4 patients (1.04\%) had evidence of NCC on MR imaging and CT. These were degenerated, calcified, or live vesicular cysticerci. One patient with headache had an intraventricular cysticercus in the fourth ventricle and other identifiable vesicular cysticerci in the brain. The difference in proportions of NCC in subjects with coinfections (19.3\%) and in control subjects (1.04\%) was statistically highly significant $(P=.0003)$.

In patients with coinfection, all 11 patients (100\%) showed JE lesions on MR imaging. JE lesions were nonenhancing and hyperintense on T2-weighted and proton attenuation images, isointense to hypointense on T1-weighted images, and were seen in the thalamus, substantia nigra, basal ganglia, hippocampi, and midbrain (Figs 1-3). In the 4 patients with coinfection who had repeat MR imaging scans, there was complete resolution of the JE lesions in 1 (Fig 1), with residual lesions in 3 patients (Fig 2). In 2 patients (patients 5 and 9), initial asymmetric JE lesions were followed by progression of the lesions on the opposite side, with symmetrical residua on follow-up (Fig 2). Nine patients (81\%) with coinfection had asymmetrical lesions in the first scan. Seven patients $(63.6 \%)$ had predominance of JE lesions on the side bearing the most NCC, 

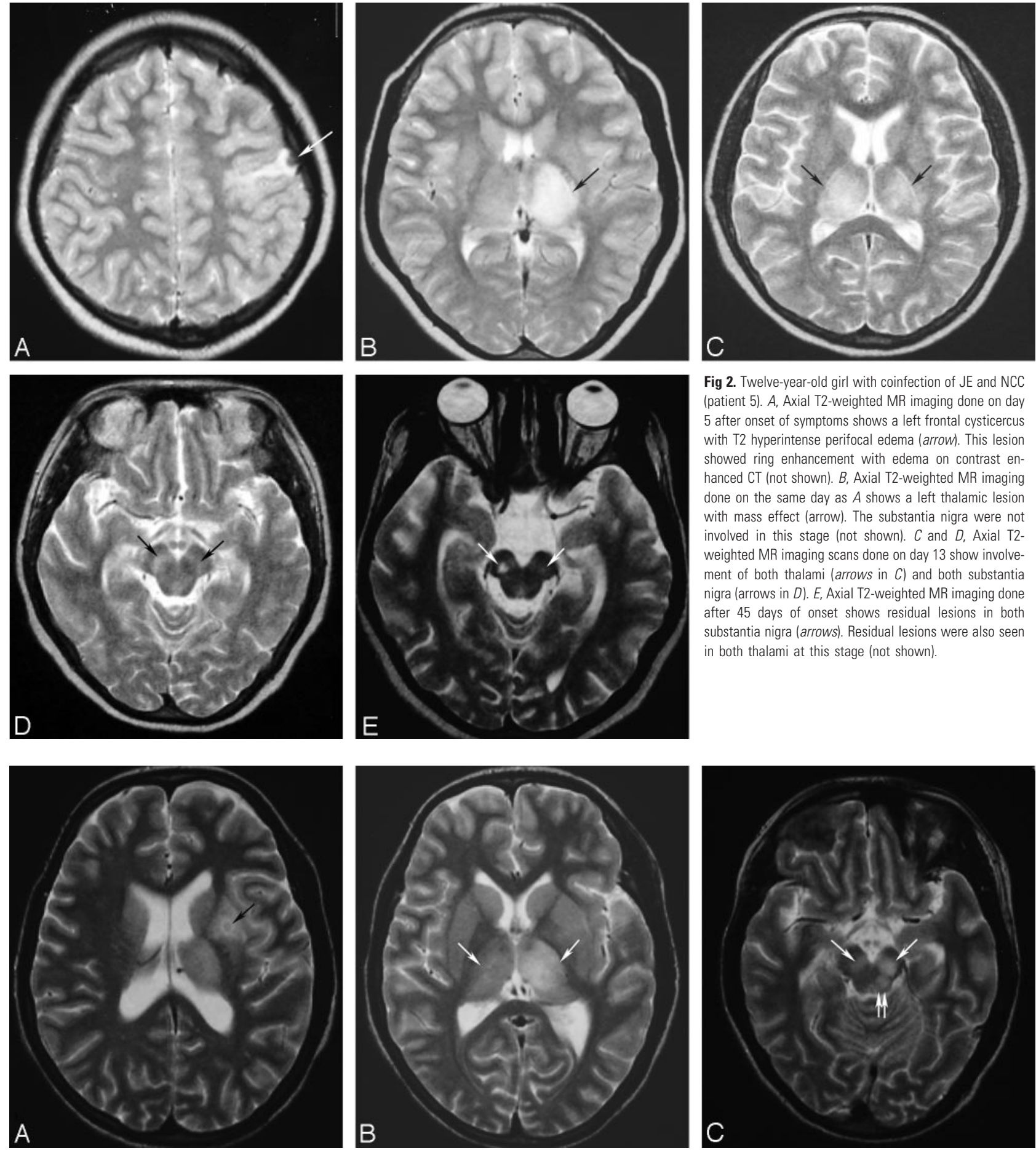

Fig 3. Thirteen-year-old girl with coinfection of JE and NCC (patient 6). A, Axial T2-weighted MR imaging scan showing a left basal ganglia granulonodular NCC with edema (arrow). $B$ Axial T2-weighted scan done at the same time as $A$ shows bilateral thalamic involvement, more on the left side (arrows). Note sparing of the basal ganglia in the vicinity of the cyst. $C$, Axial T2-weighted scan slightly lower than $B$ shows bilateral substantia nigra, left more than right (arrows) and left midbrain tectum (double arrows).

whereas 1 had predominance on the opposite side bearing the most NCC. The other 3 patients either had equal numbers of NCC on both sides or had symmetrical JE lesions with no lateralization. A significant number of asymmetric JE lesions were lateralized to the side bearing the most NCC in comparison with lateralization to the opposite side $(P=.0001)$. Eight patients showed evidence of cysts with perilesional edema (Figs 1-3). Of these, 6 had predominance of the JE lesions on the same side of the NCC with edema, 1 on the opposite side, and 1 had symmetric lesions, though statistically this predominance (6 versus 2 ) was not significant. The MR imaging findings in patients with coinfection are summarized in on-line Tables 1 and 3. CT was done in 10 patients with coinfection with plain and postcontrast CT in 9 patients. Eight (80\%) CT scans of patients with coinfection showed JE lesions. JE lesions were nonenhancing and hypoattenuated, with local mass effect in some. They were seen in the thalami, basal ganglia, and hippocampus (Fig 1). On CT, NCC was seen in 9 patients with 

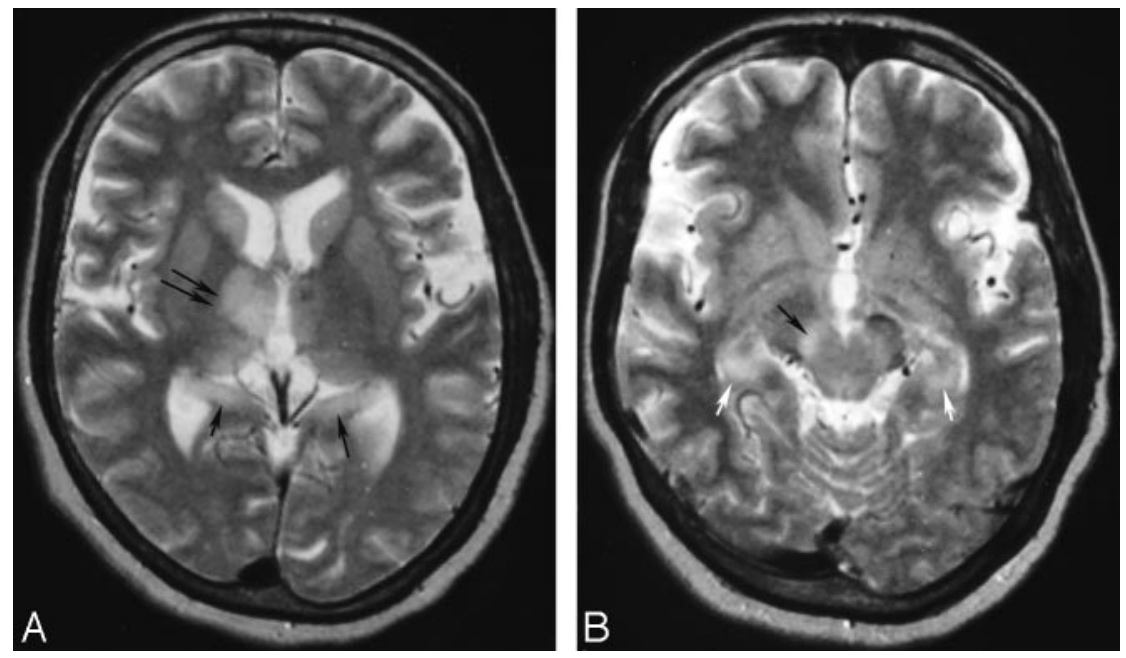

Fig 4. Fifty-eight-year-old woman with JE without NCC showing asymmetric lesions. A, Axial T2-weighted MR imaging done on day 3 of onset shows thalamic lesion on the right (double arrows). Note bilateral hippocampal tail involvement (single arrows). B, Axial T2-weighted MR imaging done on same day as $A$ shows right sided substantia nigra (black arrow) and the hippocampal lesions (white arrows). This patient is patient 2 of our previous publication. ${ }^{4}$

JE. In another retrospective study from India using MR imaging, coinfections of JE and NCC were found in $10.2 \%$. This was not statistically different from the prevalence of NCC in control subjects $(8.3 \%) .{ }^{10}$ Other imaging studies from India have not reported a high association of JE with NCC. ${ }^{2,18}$ Our study found a significantly high prevalence of NCC in patients with JE (19.3\%) in comparison with its prevalence in control subjects

coinfection and was missed in 1 because of misregistration in the emergency CT setting. The CT findings in patients with coinfection are summarized in on-line Tables 2 and 3.

In patients without coinfection, JE lesions were seen in the thalamus, substantia nigra, basal ganglia, hippocampus, cerebral cortex, midbrain, medulla, and pons on MR imaging (Fig 4) and in the thalami, substantia nigra, basal ganglia, and hippocampus on CT. The lesions were similar to those seen in patients with coinfection. No statistically significant difference was found in the distribution of JE lesions in patients with coinfection in comparison with those without coinfection on CT and MR imaging (on-line Table 3). In patients with coinfection, however, there was a significantly higher proportion of patients with asymmetrical lesions (81\%; Figs 1-3) in comparison with asymmetrical lesions in those with JE alone (45\%; $P=.0139$; Fig 4$)$. On CT, a significantly higher $(P=$ .0005) proportion of patients with coinfection showed JE lesions (80\%) in comparison with JE lesions in those without coinfections (27\%; on-line Table 3).

Some clinical parameters were compared in the patients with coinfection and those without. These were age, sex, seizures, CSF MAC-ELISA units, total leukocyte counts in the CSF, and number of deaths in hospital. Two patients were discharged on request, and the outcomes were not available. There was a significantly higher proportion of patients aged $\leq 15$ years $(P=.0329)$, and a significantly higher proportion showed CSF MAC-ELISA units $\leq 150(P=.0482)$ in the patients with coinfection. The other parameters were not significantly different between the 2 groups (on-line Table 4).

\section{Discussion}

Diffuse encephalitis with coinfection with NCC was first reported by Hsu in $1940 .{ }^{15}$ Experimental studies have shown that infecting mice with Toxocara canis, a nematode that localizes in their brains, facilitated the neuroinvasiveness of JEV, causing them to die from acute viral encephalitis. When infected with JE alone, there was very little pathogenic effect on mice. ${ }^{16,17}$ Liu et al ${ }^{8}$ reported coinfection of JE with NCC in 8 of 26 patients with JE at autopsy. Desai et al, ${ }^{6}$ using CT, serology, and autopsy, found coinfection with NCC in $37.42 \%$ of the patients with JE, which was significantly higher than the $4 \%$ autopsy prevalence of NCC in patients other than those with
$(1.04 \%)$. This high prevalence of NCC in JE is similar to the autopsy findings of some of the studies from India and China and is probably more than a chance coincidence. ${ }^{6-8}$ In autopsy studies of other infective conditions of the central nervous system (CNS), no association of NCC has been observed. ${ }^{6}$ Why other investigators from India have not found a significant association between the 2 infections is intriguing. ${ }^{2,10,18}$ It may be related to different strains of the JEV, other epidemiologic factors, or study designs. Autopsy studies have shown the prevalence of NCC in India to be $0.75 \%-4.00 \%{ }^{6,19}$ The prevalence of NCC in our control group is comparable with this. Although the diagnosis of NCC in our study was made from neuroimaging findings alone, and CSF ELISA tests for NCC done in 4 of our patients with coinfection was negative, ELISA tests are known to be disappointing in sensitivity and specificity for NCC in routine clinical and epidemiologic use. ${ }^{20}$ Imaging alone is considered adequate for the diagnosis of NCC in our institute.

In patients with coinfections, lesions of JE tended to be asymmetrical with a significantly higher proportion of asymmetrical JE lesions in coinfections in comparison to those without coinfections. There was significant lateralization of JE lesions to the side bearing the most NCC, and a greater number of JE lesions were seen on the side with the most NCC with edema. Singh et al, ${ }^{9}$ in an MR imaging-based study, found asymmetric thalamic lesions in all 10 of their patients with coinfection. JE lesions were lateralized to the side bearing the most NCC or to the side bearing the edematous NCC. Desai et $\mathrm{al}^{6}$ found that JE lesions were more severe on the side bearing the NCC with host reaction. Our findings were similar to these reports.

On imaging, the JE lesions in coinfection were not different in appearance or distribution compared with the lesions seen in those without coinfections in our study. However, a significantly higher number of JE lesions on CT and a higher number of lesions on MR imaging, though not statistically significant, suggest more severe lesions of JE in patients with coinfections compared with those without. JE lesions are not commonly picked up on CT, and a little more than one third of patients with JE showed abnormal scans in one study. ${ }^{18}$ Two types of pathologic lesions are noted in JE-infected brains, the cell-rich gliomesenchymal nodules (GMNs) and areas of rar- 
efaction necrosis, the necrolytic lesions (NLs). The lesions are seen in the in the thalami, substantia nigra, basal ganglia, pons, medulla, cerebellum, and cerebral cortex, explaining the distribution of JE lesions on imaging. Differentiation of the 2 types of lesions on imaging is not possible. Most of the affected areas show both types of lesions pathologically. NLs are, however, uncommon in the medulla, whereas GMNs are invariably seen in the medullary inferior olivary nucleus. ${ }^{7}$ Considering the rarity of demonstrable lesions in the medulla in our study, as well as those of others, ${ }^{2}$ it is possible that the NL is the predominant lesion picked up on imaging. Pathologically, more florid JE lesions with predominance of NLs are seen in patients with coinfections. ${ }^{6,7}$ This may explain the higher number of JE lesions seen in coinfections on imaging in our study. However, we were unable to do any autopsies in these patients to confirm our findings.

The more florid JE lesions seen on imaging in coinfections with NCC in comparison with those without and lateralization of the lesions to the side bearing the most NCC or the edematous NCC suggest increased neuroinvasiveness of JEV, possibly resulting from a synergistic effect between the 2 infections. The JEV invades the brain by the hematogenous route through the blood-brain barrier (BBB). ${ }^{21}$ Increased BBB permeability with increased neuroinvasiveness of JEV has been advocated in coinfections of JE with NCC. ${ }^{16,17}$ The reasons for the subsequent involvement of the thalami and other preferred sites may be because of JEV-specific susceptible neurons. ${ }^{21}$ The distribution of JE lesions in patients with coinfections in our study, as well as in others, was not different from those seen in patients of JE only, other than severity and lateralization to the side having the most or degenerating NCC. ${ }^{6,9}$ This suggests that the anatomic sites of BBB leakage or neuronal susceptibility to JEV in coinfections are possibly not different from those seen in patients of JE alone and are not in the vicinity of the cyst, which would have resulted in florid JE lesions around it. In mice models of NCC, there is increased $\mathrm{BBB}$ permeability, but the $\mathrm{BBB}$ in the vicinity of the cyst is not altered, and differences in the extent and timing of permeability of different types of vessels have been demonstrated. ${ }^{22}$ NCC, therefore, possibly potentiates neuroinvasiveness and virulence of the JEV rather than altering the anatomic sites of JEV invasion or neuronal susceptibility to JEV. Progression of JE lesions in 2 of our patients with coinfection suggests increased BBB permeability to JEV neuroinvasion or even a temporal variation of $\mathrm{BBB}$ permeability in these patients.

Another finding in our study was the significantly large number of patients with coinfection who showed low CSF MAC-ELISA units in comparison with patients with JE only reflecting lower CSF IgM levels to JEV in these patients. CSF IgM levels show a temporal variation throughout the course of JE. They peak by day 7 of onset and persist up to day 30, decreasing thereafter. ${ }^{11}$ This temporal variation was unlikely to have affected our results, because there was no significant difference in the sampling times of the CSF in the patients with coinfection versus those without (on-line Table 5). In a study in mice models of dual infection that showed potentiation of JEV pathogenicity, an altered immune response and a delayed peak of IgM levels in CSF by 7 days was observed. ${ }^{23}$ The only other study that evaluated MAC-ELISA levels in dual infections did not find lowered IgM levels in their patients with coinfection. However, they had used higher cutoff levels of CSF MAC-ELISA units (500 U). ${ }^{6}$ Our patients showed lower MAC-ELISA units that varied from 100 to $315 \mathrm{U}$. Whether altered IgM kinetics in the CSF or a suppressed host immune response was responsible for the low CSF IgM levels is uncertain and needs further evaluation. An altered host immune response in patients with coinfection could be a factor in the synergistic effect between the 2 infections.

Patients with coinfections were significantly younger than those with JE alone in our study. Even in endemic regions, NCC is not common in children. Hospital-based data indicate that the frequency of NCC below the age of 15 years is approximately one tenth the frequency above that age. This is possibly due to the fact that pediatric patients are protected from environmental factors that cause NCC. ${ }^{24}$ The high proportion of pediatric patients with coinfections is, therefore, interesting. Although Desai et $\mathrm{al}^{6}$ did not find higher coinfections in children, Kalita et $\mathrm{al}^{18}$ found all 3 of the coinfections that they saw in children. The overall pediatric patients in our study were 16 (25\%), a proportion much less than the ratio of pediatric-toadult JE reported from Phukan et $\mathrm{al}^{25}$ at 1.4:1 during 20002002. Our patients were from a diverse rural, semirural, and urban background and did not belong to the low socioeconomic group, unlike those of other studies from India with which our data are compared. ${ }^{6,9,25}$ This may have caused a hospital-based data bias. Nevertheless, coinfections of JE and NCC may still be more common in children due to immunologic factors, an immature CNS, or neuronal plasticity, factors that have been implicated for JE being more common in children. ${ }^{18}$

Coinfection with NCC resulted in significantly increased deaths from JE in the study by Desai et al. ${ }^{6}$ Although we did see a higher proportion of hospital deaths in coinfections (25\%) compared with those subjects with JE only (14.5\%), this difference was not statistically significant. We did not, however, score the outcomes in our survivors. The overall deaths (10 of $60[16.6 \%])$ in our study were far fewer than the death rates of more than 40\% reported from Kabilan et al in 2000 and $2001 .^{1}$ This was probably due to the fact that our patients were treated in a well-equipped neurointensive care unit.

In patients clinically suspected of acute encephalitis, the presence of an enhancing NCC may divert attention away from associated JE. The reverse is also true, with the possibility of NCC being missed on imaging or misinterpreted as lesions of JE. This is also further compounded by the fact that encephalitic presentation of NCC is possible. Cysticercus encephalitis is a syndrome of heavy multilesional NCC with increased intracranial pressure. This condition is not seen with single or few NCC, the types more common in India. Confluent brain edema with enhancing lesions typical of NCC is seen on imaging. ${ }^{26}$ We have not seen JE in patients with heavy multilesional NCC. In the usual situation, a careful evaluation of the brain for JE lesions in clinically suspected patients with acute encephalitis and conscious evaluation for NCC on imaging can help pick up patients with coinfection.

\section{Conclusion}

In conclusion, the high prevalence of NCC in JE is probably more than a chance occurrence. Although there was no significant difference in the anatomic distribution of JE lesions in the brain between the group with coinfection and that without 
coinfection, NCC seems to have a synergistic effect on JE facilitating the entry of the virus into the brain, causing more florid JE lesions on the ipsilateral side of the maximal and/or degenerating NCC as shown by the imaging findings. An alteration in the immune response in coinfections is suggested by the lowered levels of the CSF JEV-specific IgM observed in this study. Children may be particularly susceptible to coinfections. Further prospective and experimental studies are necessary to clarify the complex epidemiologic, pathologic, and immunologic factors in coinfections. The synergistic effect of NCC in JE suggests the possibility of a need for control of NCC in addition to vector control, vaccination, and other measures in the control of the spread of JE.

\section{Acknowledgments}

We thank Nupur Choudhury for her help in statistical analysis.

\section{References}

1. Kabilan L, Rajendran R, Arunachalam N, et al. Japanese encephalitis in India: an overview. Indian J Paediatr 2004;71:609-15

2. Kalita J, Misra UK. Comparision of CT scan and MRI findings in the diagnosis of Japanese encephalitis. J Neurol Sci 2000;174:3-8

3. Shoji $\mathrm{H}, \mathrm{Kida} \mathrm{H}, \mathrm{Hino} \mathrm{H}$, et al. Magnetic resonance imaging findings in Japanese encephalitis. White matter lesions. J Neuroimaging 1994;4:206-11

4. Handique SK, Das RR, Barman K, et al. Temporal lobe involvement in Japanese encephalitis-problems in differential diagnosis. AJNR Am J Neuroradiol 2006; 27:1027-31

5. Del Brutto OH, Rajshekhar V, White AC Jr, et al. Proposed diagnostic criteria for neurocysticercosis. Neurology 2001;57:177-83

6. Desai A, Shankar SK, Jayakumar PN, et al. Co-existence of cerebral cysticercosis with Japanese encephalitis: a prognostic modulator. Epidemiol Infect 1997;118:165-71

7. Shankar SK, Vasudev Rao T, Mruthyunjayanna BP, et al. Autopsy study of brains during an epidemic of Japanese encephalitis in Karnataka. Ind J Med Res 1983;78:431-40

8. Liu YF, Teng CL, Liu K. Cerebral cysticercosis as a factor aggravating Japanese encephalitis. Chin Med J 1957;75:1010-16

9. Singh P, Kalra N, Ratho RK, et al. Co-existent neurocysticercosis and Japanese B encephalitis: MR imaging co-relation. AJNR Am J Neuroradiol 2001;22:1131-36
10. Azad R, Gupta RK, Kumar S, et al. Is neurocysticercosis a risk factor in coexistent intracranial disease? An MRI based study. J Neurol Neurosurg Psychiatry 2003;74:359-61

11. Burke DS, Nisalak A, Ussery MA, et al. Kinetics of IgM and IgG responses to Japanese encephalitis virus in human serum and cerebrospinal fluid. J Infect Dis 1985;151:1093-99

12. Osborn AG. Infections of the brain and its linings. In: Osborn AG, ed. Diagnostic Neuroradiology. St Louis: Mosby - Year Book; 1994:673-715

13. Udani V. Pediatric epilepsy — an Indian perspective. Indian J Pediatr 2005;72: 309-13

14. Singh G, Singh P, Singh I, et al. Epidemiological classification of seizures associated with neurocysticercosis; observations from a sample of seizure disorders in neurologic care in India. Acta Neurol Scand 2006;113:233-40

15. Hsu YK. Cerebral cysticercosis and acute poliomyeloencephalitis. Chinese Med J 1940;57:318-19

16. Mochizuki $\mathrm{H}$, Tomimura $\mathrm{T}$, Takenori $\mathrm{O}$. Cerebral nematodiasis as a provoking factor in Japanese B encephalitis: an experimental approach. J Infectious Dis 1954;95:260-66

17. Pavri KM, Ghalsasi GR, Dastur DK, et al. Dual infections of mice: visceral larva migrans and sublethal infection with Japanese encephalitis virus. Trans $R$ Soc Trop Med Hyg 1975;69:99-110

18. Kalita J, Misra UK, Pandey S, et al. A comparision of clinical and radiological findings in adults and children with Japanese encephalitis. Arch Neurol 2003;60:1760-64

19. Singh G, Prabhakar S, Ito A, et al. Taenia solium taeniasis and cysticercosis in Asia. In: Singh G, Prabhakar S, eds. Taenia Solium Cysticercosis. Oxon, United Kingdom: CAB International; 2002:111-27

20. Pal DK, Carpio A, Sander JWAS. Neurocysticercosis and epilepsy in developing countries. J Neurol Neurosurg Psychiatry 2000;68:137-43

21. Tiroumourougane SV, Raghava P, Srinivasan S. Japanese viral encephalitis. Postgrad Med J 2002;78:205-15

22. Alvarez JI, Teale JM. Breakdown of the blood brain barrier and the bloodcerebrospinal fluid barrier is associated with differential leukocyte migration in distinct compartments of the CNS during the course of murine NCC. J Neuroimmunol 2006;173:45-55

23. Gupta AK, Pavri KM. Alteration in immune response of mice with dual infection of Toxocara canis and Japanese encephalitis virus. Trans $R$ Soc Trop Med Hyg 1987;81:835-40

24. Prabhakar S, Singh G. Paediatric neurocysticercosis. In: Singh G, Prabhakar S, eds. Taenia Solium Cysticercosis. Oxon, United Kingdom: CAB International; 2002:257-62

25. Phukan AC, Borah PK, Mahanta J. Japanese encephalitis in Assam, northeast India. Southeast Asian J Trop Med Public Health 2004;35:618-22

26. Del Brutto OH, Garcia HH, Prabhakar S. Heavy multilesional cysticercotic syndromes. In: Singh G, Prabhakar S, eds. Taenia Solium Cysticercosis. Oxon, United Kingdom: CAB International; 2002:189-97 